Research paper

\title{
Infection of hematopoietic stem cells by Leishmania infantum increases erythropoiesis and alters the phenotypic and functional profiles of progeny
}

\author{
Raquel Carvalho-Gontijo ${ }^{\mathrm{a}}$, Diana Raquel Moreira ${ }^{\mathrm{b}, \mathrm{c}}$, Mariana Resende, ${ }^{\mathrm{b}, \mathrm{e}}$, \\ Matheus Fernandes Costa-Silva ${ }^{\mathrm{a}}$, Vanessa Peruhype-Magalhães ${ }^{\mathrm{a}}$, Cláudia Maria Franco Ribeiro ${ }^{\mathrm{d}}$, \\ Daniel Dias Ribeiro $^{\mathrm{d}}$, Ricardo Silvestre ${ }^{\mathrm{e}}$, Anabela Cordeiro-da-Silva ${ }^{\mathrm{b}, \mathrm{c}, \mathrm{f}}$, \\ Olindo Assis Martins-Filho ${ }^{\mathrm{a}}$, Andréa Teixeira-Carvalho ${ }^{\mathrm{a}, *}$ \\ a Grupo Integrado de Pesquisa em Biomarcadores, Instituto René Rachou, FIOCRUZ, Belo Horizonte, Brazil \\ ${ }^{\mathrm{b}}$ Instituto de Biologia Molecular e Celular, Porto, Portugal \\ ${ }^{\mathrm{c}}$ I3S - Instituto de Investigação e Inovação em Saúde, Porto, Portugal \\ d Clínica Hematológica, Belo Horizonte, Brazil \\ ${ }^{\mathrm{e}}$ Life and Health Sciences Research Institute (ICVS), School of Medicine, University of Minho, Braga, Portugal and ICVS/3B's-PT Government Associate Laboratory, \\ Braga/Guimarães, Portugal \\ ${ }^{\mathrm{f}}$ Faculdade de Farmácia, Universidade do Porto, Portugal
}

\section{A R T I C L E I N F O}

\section{Keywords:}

Stem cells

Leishmania

Hematopoiesis

Hematopoietic progeny

Immunophenotyping

Cytokines

\begin{abstract}
A B S T R A C T
Immunosuppression is a well-established risk factor for Visceral Leishmaniasis. Post-immunosuppression leishmaniasis is characterized by an increase of parasite burden, hematopoietic disorders and unusual clinical manifestations. Although there are many reports on bone marrow findings in VL, less is known about the relationship between parasite dynamics in this organ and the function of either hematopoietic stem cells and progenitor cells themselves. In the present study, we tackle these issues using a new approach of infecting human stem cells derived from bone marrow with $L$. infantum. Using this strategy, we show that human hematopoietic stem cells (hHSC) are able to phagocytize L. infantum promastigotes and release modulatory and pro-inflammatory cytokines, mainly TNF- $\alpha$. Our results demonstrated that L. infantum infection in vitro enhances hematopoiesis, favoring the development of erythrocitic lineage through a mechanism yet unknown. Moreover, we found that $L$. infantum infection alters the phenotypic profile of the hematopoietic progeny; modifying the surface markers expression of differentiated cells. Thus, our study represents a rare opportunity to monitor the in vitro differentiation of human stem cells experimentally infected by $L$. infantum to better understand the consequences of the infection on phenotypic and functional profile of the cell progeny.
\end{abstract}

\section{Introduction}

Leishmaniasis is a group of diseases caused by kinetoplastids belonging to the genus Leishmania, which are transmitted by sandfly bites during a blood meal. Leishmania sp. causes a wide spectrum of human diseases, ranging from being asymptomatic to lethal visceral leishmaniasis [2,21]. Whereas most immunocompetent individuals will not develop disease after Leishmania infection, immunosuppression is a well-established risk factor for disease. Although immunosuppression has historically been mainly observed in human immunodeficiency virus (HIV-infected patients), non-HIV related immunosuppressive conditions are becoming more common, mainly because of the therapeutic use of immunosuppressive drugs [27]. Some previous research has been attracting attention for documenting atypical clinical manifestations in individuals diagnosed with leishmaniasis, after use of immunosuppressive drugs $[8,11,12]$. Clinical presentation in immunosuppressed individuals is characterized by an increase in parasite burden, hematopoietic disorders and atypical clinical manifestations, which are usually misdiagnosed or accepted as a flare-up of an unrelated underlying condition. The absence of clinical signals or symptoms before immunosuppression suggests that Leishmania parasites are using sites of immunological privilege, such as bone marrow cells, for maintenance and multiplication without disease manifestation under normal immunological conditions [11]. Furthermore, the presence of Leishmania donovani amastigotes inside bone marrow hematopoietic stem cells and stromal macrophages has been reported after

\footnotetext{
* Corresponding author

E-mail address: andreat@minas.fiocruz.br (A. Teixeira-Carvalho).
} 
experimental infection of Balb/c mice by Cotterell et al. [7].

Few studies have addressed the immunological consequences of bone marrow infection by visceral Leishmania spp. Leishmania-infected stromal cells display a modified capacity to recruit and support hematopoietic progenitor differentiation into regulatory DCs. Moreover, aberrant expression of CCL8 by diseased stromal tissue may be involved in the switch from resolving to persistent infection [18]. Besides, it has been reported that $L$. donovani infection of stromal macrophages enhances myelopoiesis trough a mechanism involving GM-CSF and TNF- $\alpha$ [7].

Thus, to better understand the particularities that are involved in this type of infection, we infect stem cells derived from human bone marrow with $L$. infantum promastigotes. Using this strategy, we show that human hematopoietic stem cells are able to phagocytize $L$. infantum promastigotes and release pro-inflammatory and modulatory cytokines early after infection. Moreover, our results indicated that infection by $L$. infantum promotes an increase of red and white colonies after in vitro hematopoiesis assay through a yet unknown mechanism. Thus, our study represents a rare opportunity to monitor the in vitro differentiation of human stem cells infected by $L$. infantum and understand the impact on the phenotypic and functional immune profile of their progeny (Table 1).

\section{Samples, material and methods}

\subsection{Samples}

Human hematopoietic stem cells (hHSC) used in this study were obtained from Hematological Clinic, Belo Horizonte, Brazil. All donors who agreed to collaborate with this study signed an informed written consent form. These patients were carriers of hematological diseases that do not compromise the medullary compartment. All donors were monitored by a hematologist to ensure the eligibility of samples. The HSCs were collected by leukapheresis after the use of the drug G-CSF by the donors, which stimulate the proliferation and mobilization of these cells to the peripheral blood. After collection, these cells were cryopreserved at $-80{ }^{\circ} \mathrm{C}$, using a commercial solution of hydroxyethyl starch solution $60 \%$ (HES), albumin $40 \%$ and dymethilsulfoxide $10 \%$ (DMSO). All the donors, carriers of hematological diseases, were successfully transplanted with autologous cells and cured after the process, guaranteeing the quality of the collected samples. The cells used in the present study are those that would be discarded by the clinic because of loss of shelf life for use in human medical procedures. The use of this type of biological material has been validated by the Agência Nacional de Vigilância Sanitária (National Sanitary Surveillance Agency of Brazil ANVISA). All the experiments were performed using representative

Table 1

Samples donnors.

\begin{tabular}{llllll}
\hline ID & Sex & Age & Diagnosis & $\%$ CD34 & LGC $/ \mathrm{mm}^{3}$ \\
\hline RCPP & M & 55 & MM & 0.20 & 313,750 \\
WBJ & M & 51 & MM & 0.19 & 318,750 \\
VAS & F & 52 & MM & 0.17 & 297,500 \\
RFS & M & 46 & MM & 0.27 & 314,500 \\
AJS & M & 40 & MM & 7.23 & 242,500 \\
NJP & F & 45 & MM & 0.45 & 278,400 \\
JMC & M & 48 & H & 0.56 & 280,200 \\
DNPS & F & 42 & H & 0.22 & 256,300 \\
RAF & M & 55 & MM & 0.22 & 260,000 \\
JFFA & F & 50 & HL & 2.09 & 278,250 \\
MCCO & F & 66 & MM & 0.18 & 162,500 \\
MALM & F & 73 & MM & 0.45 & 232,500 \\
OGA & M & & & 0.70 & 303,250 \\
ACGS & M & 61 & MM & 1.06 & 232,250 \\
\hline
\end{tabular}

M- Male, F- Female, MM- Multiple Myeloma, H- Healthy, HL- Hodgkin's lymphoma, GCLeukocyte's global concentration samples of healthy and baseline diseases donors. This work complied with resolution number 466/2012 from the National Health Council for research involving human subjects and was approved by the Ethical Committee at Instituto René Rachou (CEPSH/IRR/FIOCRUZ protocol 1.368.058), Belo Horizonte, Minas Gerais, Brazil.

\subsection{Viability of hematopoietic stem cells (hHSC)}

The viability of all samples was evaluated using Trypan Blue labeling. Live and dead cells were counted using an automated cell counter (Countess ${ }^{\mathrm{TM}}$ - Thermo Scientific) to assess viability percentage. The ideal viability for the use of cells had to be equal to or higher to $85 \%$.

\subsection{Alexa Fluor 647-labeling procedure for L. Infantum}

Promastigote forms of L. infantum - PP75 strain - were cultivated in Schneider's Drosophila Medium (Thermo Fischer ${ }^{\mathrm{TM}}$ ) supplemented with $20 \%$ of fetal bovine serum (FBS - Sigma Aldrich ${ }^{\mathrm{TM}}$ ) and NNN (Novy, MacNeal, Nicolle) medium for 8 days at $25^{\circ} \mathrm{C}$ in a B.O.D incubator. Live and fixed parasites were stained with Alexa Fluor 647 (AF647 - NHS Ester - Thermo Fischer ${ }^{\mathrm{TM}}$ ) at a final concentration of $3.2 \mu \mathrm{g} / \mathrm{mL}$ at $37^{\circ} \mathrm{C}$ for $30 \mathrm{~min}$ in a $5 \% \mathrm{CO}_{2}$ humidified incubator. After the labeling procedure, parasites were washed three times with PBS $1 \mathrm{x}$ and re-suspended in PBS $1 \mathrm{x}$ supplemented with $10 \%$ FBS. The AF647-labeled parasite suspension was adjusted to $1 \times 10^{8} / \mathrm{mL}$, and maintained at $25^{\circ} \mathrm{C}$ in a B.O.D. incubator until use. For the fixed $L$. infantum condition, after the third wash step, parasites were re-suspended in FACS fix solution $10.0 \mathrm{~g} / \mathrm{L}$ of paraformaldehyde, $1 \%$ sodium cacodylate, $6.63 \mathrm{~g} / \mathrm{L}$ of sodium chloride and $0.01 \%$ of sodium azide -Sigma Chemical- $\mathrm{pH}$ 7.2) and stored at $4{ }^{\circ} \mathrm{C}$ until use as described by Vitelli-Avelar et al. [28]. Aliquots of AF647-labeled parasites were then run through a flow cytometer to evaluate the efficiency of the AF647 staining procedure. Ideal AF647-labeled parasite staining would lead to a single peak around $10^{2}$ and $10^{3}$ log intervals in FL4/AF647 histogram plots. Additionally, the live-labeled parasites were monitored by optical microscopy to assess motility and, via Trypan blue staining, viability quality control.

\subsection{Short-term in vitro phagocytosis protocol with AF647-labeled live and fixed L. Infantum}

To perform the culture of hHSC, the number of cells was adjusted to $1 \times 10^{7}$ cells/mL using RPMI- $1640\left(\mathrm{GIBCO}^{\mathrm{TM}}\right)$ supplemented with $0.5 \%$ of sodium heparin. Short-term in vitro cultures were performed using $14 \mathrm{~mL}$ polypropylene tubes (Falcon ${ }^{\mathrm{Tu}}$; BD Pharmingen, San Jose, CA, USA) in three distinct platforms referred to as "Unstimulated (CT)", "Live $L$. infantum-Stimulated (Leish-L)" and "Fixed $L$. infantumStimulated (Leish-F)" cultures. For "CT" cultures aliquots of $1 \mathrm{~mL}$ hHSC suspension were incubated with $1 \mathrm{~mL}$ of RPMI- 1640. For the Leish-L cultures, aliquots of $1 \mathrm{~mL}$ of hHSC suspension were incubated with $500 \mu \mathrm{L}$ of RPMI-1640 and $500 \mu \mathrm{L}$ of AF647-labeled live L. infantum organisms at $1 \times 10^{8}$ parasites $/ \mathrm{mL}$. For the Leish-F cultures aliquots of $1 \mathrm{~mL}$ of hHSC suspension were incubated with $500 \mu \mathrm{L}$ of RPMI-1640 and $500 \mu \mathrm{L}$ of AF647-labeled fixed L. infantum organisms at $1 \times 10^{8}$ parasites/mL.

Both, unstimulated and stimulated cultures were performed in duplicate. The tubes were incubated under gentle shaking by an orbital shaker for $120 \mathrm{~min}$ at $37{ }^{\circ} \mathrm{C}$ in a $5 \% \mathrm{CO}_{2}$ humidified incubator. Following the incubation, the cultures were treated with EDTA (Ethylenediamine tetraacetic acid - SIGMA ${ }^{\mathrm{TM}}$ ) at $20 \mathrm{mM}$, and maintained at room temperature for $15 \mathrm{~min}$ prior to immunophenotypic staining for cell surface markers. 
A

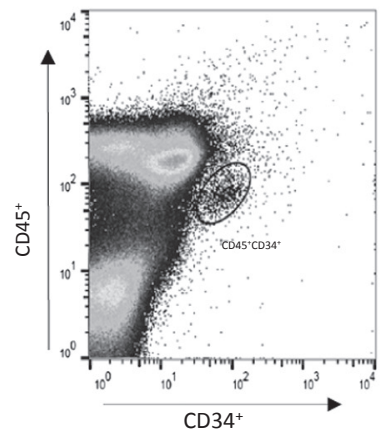

B

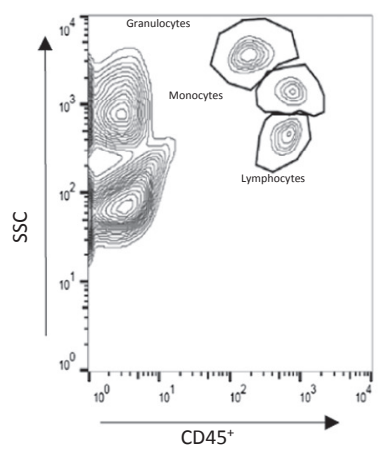

C
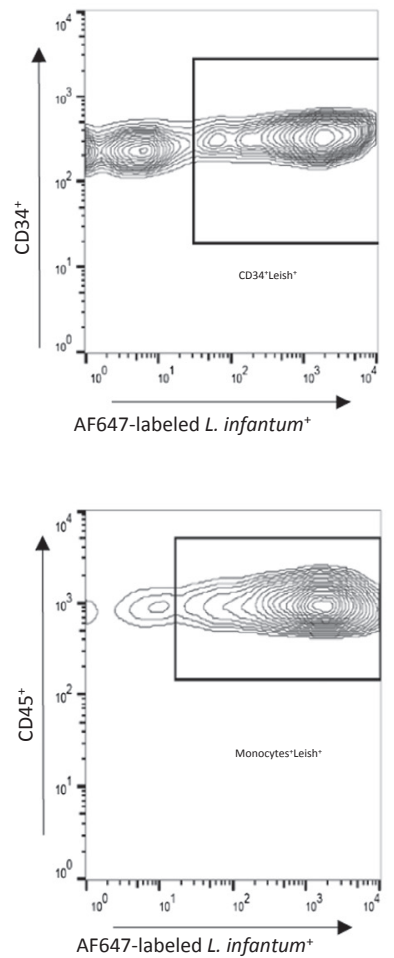

$\mathbf{E}$


D

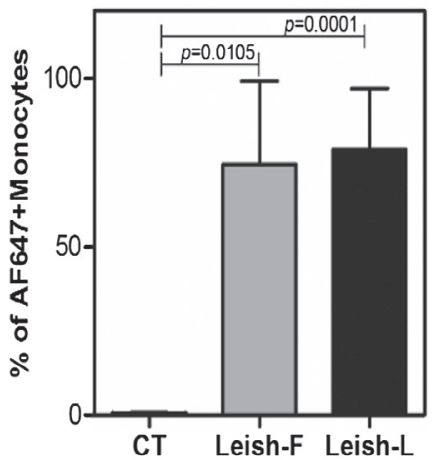

$\mathbf{F}$

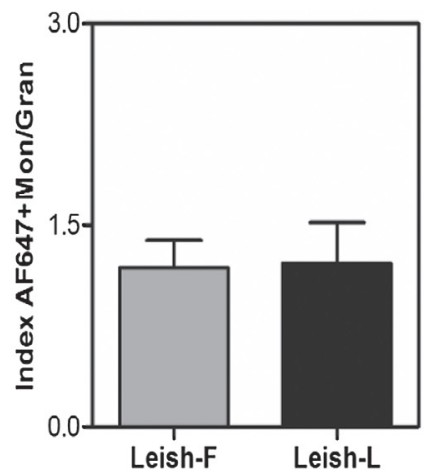

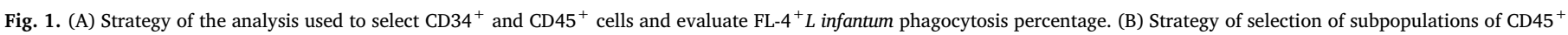

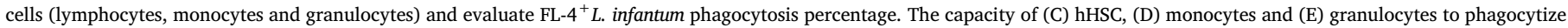

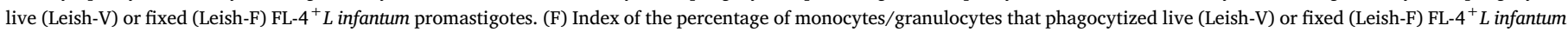

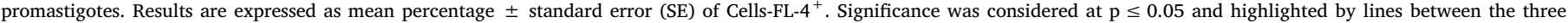
distinct groups.

\subsection{Analysis of cell surface markers and intracytoplasmic cytokines}

For cell surface marker analysis, aliquots of $200 \mu \mathrm{L}$ of cell cultures obtained from the phagocytosis assay were incubated for $30 \mathrm{~min}$ at room temperature with (PerCP)-labeled anti-CD34 mAb (clone 8G12), (FITC)-labeled anti-CD45 mAb (clone 2D1), (FITC)-labeled anti-CD33 (clone HIM3-4), (PE)-labeled anti-CD13 (clone WM15), (FITC)-labeled anti-HLA-DR mAb (clone G46-6), (PE)-labeled anti-CD14 mAb (clone M5E2), (PE)-labeled anti-CD41a mAb (clone HIP8), (FITC)-labeled antiCD235a mAb (clone GA-R2) and (FITC)-labeled anti-CD19 mAb (clone HIB19), all purchased from Becton Dickinson-BD ${ }^{\mathrm{TM}}$. Following incubation, the red blood cells were lysed with FACS lysing solution (BD Biosciences $^{\mathrm{TM}}$ ), except the samples labeled with FITC-anti-CD235a, which identifies erythrocyte lineage. The samples were washed with FACS buffer (PBS $0.5 \%$ of bovine serum albumin and $0.1 \%$ sodium azide - BD Biosciences ${ }^{\mathrm{TM}}$ ), and fixed with FACS fix solution. A total of 50,000 events were acquired in a FACScalibur flow cytometer (Becton Dickinson and Company ${ }^{\mathrm{TM}}$ ), and analyses of the immunophenotypic and morphometric parameters were determined using FlowJo ${ }^{\mathrm{TM}}$ version 10.07 .

For intracytoplasmic cytokine analysis, aliquots of $500 \mu \mathrm{L}$ of cell cultures were incubated for $30 \mathrm{~min}$ at room temperature with PerCPlabeled anti-CD34 and FITC-labeled anti-CD45 mAbs to identify hHSC subsets. Following incubation, the red blood cells were lysed with FACS lysing solution and the samples were kept with FACS permeabilizing solution. Following incubation, the samples were centrifuged at $600 \times g$ for $7 \mathrm{~min}$ at room temperature and the cell pellet washed with FACS buffer. After centrifugation, the cells were resuspended with FACS buffer. Cells were then stained with anti-cytokine mAbs including PElabeled anti-IFN- $\gamma$ (clone B27), anti-IL-10 (clone JES3-19F1) anti-IL-4 (clone 8D4-8), anti-TNF- $\alpha$ (clone MAb11) by incubation for $30 \mathrm{~min}$ at room temperature in the dark. After incubation, the cells were washed twice and fixed with FACS fix solution. A total of 50,000 events were acquired in a FACScalibur flow cytometer and morphometric, phenotypic and functional parameters were determined using FlowJo ${ }^{\mathrm{TM}}$ version 10.7.

\subsection{In vitro hematopoiesis assay}

A total of $5 \times 10^{6}$ cells in the presence (Leish-F and Leish-L) or absence (CT) of $L$. infantum stimuli was placed in six-well plates with MethoCult $\mathrm{H} 4034$ (Stem Cell $^{\mathrm{TM}}$ ), a specific medium containing all growth factors necessary to perform hematopoiesis in vitro. The cell suspension was incubated for fourteen days at $37^{\circ} \mathrm{C}$ in a $\mathrm{CO}_{2}$ incubator. At the endpoint of the cultures, the colonies formed were counted and photographed using an inverted microscope coupled to a camera (Axio Vert.A1 microscope, Zeiss ${ }^{\mathrm{TM}}$ ). For phenotypic analysis by flow cytometry, the colonies, both red and white, were recovered from the sixwell plates, removed from culture medium and washed with FACS buffer. For cell surface marker analysis, the cell suspensions were incubated for $30 \mathrm{~min}$ at room temperature with PerCP-labeled anti-CD34 $\mathrm{mAb}$ and (FITC)-labeled anti-CD45 mAb, (FITC)-labeled anti-CD33, (PE)-labeled anti-CD13, (FITC)-labeled anti-HLA-DR mAb, (PE)-labeled anti-CD14 mAb, CD41a mAb, (FITC)-labeled anti-CD235a mAb and (FITC)-labeled anti-CD19 mAb, all purchased from Becton Dickinson$\mathrm{BD}^{\mathrm{TM}}$. The samples were washed with FACS buffer, and fixed with FACS FIX solution. A total of 50,000 events were acquired in a FACScalibur flow cytometer, and analyses of the immunophenotypic and morphometric parameters were determined using Flow $\mathrm{Jo}^{\mathrm{TM}}$ software (version 10.07). 


\section{Results}

3.1. Bone marrow-derived hHSC, monocytes and granulocytes are able to phagocytize L. Infantum promastigotes

We started to evaluate the capacity of bone marrow-derived hHSC, monocytes and granulocytes to phagocytize live or fixed $L$. infantum promastigotes. Upon two hours of interaction, we observed that hHSCs phagocyted a high percentage of both live as well as fixed $L$. infantum when compared with the CT group (Fig. 1C). Similarly, we observed a high percentage of monocytes and granulocytes that internalized fixed (Leish-F) and live (Leish-L) L. infantum promastigotes in comparison to the CT group (Fig. 1D and E). Aiming to compare the phagocytic capacity between monocytes and granulocytes, we calculated an index of the percentage of monocytes FL- $4^{+}$divided by granulocytes FL- $4^{+}$and found that about 1.5 times more monocytes phagocytized live or fixed L. infantum than granulocytes (Fig. 1F).

\subsection{Bone marrow-derived hHSC, lymphocytes, monocytes and granulocytes} produce high levels of modulatory and pro-inflammatory cytokines, especially TNF- $\alpha$, after L. Infantum internalization

Aiming to evaluate the functional profile of hHSC, lymphocytes, monocytes and granulocytes derived from bone marrow, we analyzed the intracytoplasmic expression of IL- 4 , IL-10, IFN- $\gamma$ and TNF- $\alpha$, after stimulation with fixed or live $L$. infantum. The expression of these cytokines are crucial for disease evolution, the balance between pro-inflammatory and modulatory is related to a good prognosis. Data analysis demonstrated an increased frequency of hHSC IL- $4^{+}$when we compared Leish-L or Leish-F with non-infected cultures (Fig. 2C); the same significant increase of granulocytes, lymphocytes and monocytes $\mathrm{IL}-4^{+}$was observed comparing Leish-L with Leish-F or CT group (Fig. 3C). Evaluating another modulatory cytokine expression, we observed a high percentage of hHSC IL- $10^{+}$when we compared Leish-L with Leish-F with the CT group (Fig. 2D). Moreover, we found a significant increase in the percentage of lymphocytes, monocytes and granulocytes IL- $10^{+}$comparing Leish-L and Leish-L with the CT group (Fig. 3D). Analysis of pro-inflammatory cytokine production demonstrated a high percentage of hHSC IFN- $\gamma^{+}$when we compared Leish-L and Leish-F with the CT group (Fig. 2E). The same pattern occurs when we analyze IFN- $\gamma^{+}$production by lymphocytes, monocytes and granulocytes derived from bone marrow (Fig. 3E). Finally, we evaluated TNF$\alpha$ production and observed a great increase in the percentage of hHSC TNF- $\alpha$ producers when comparing Leish-L with Leish-F with the CT group (Fig. 2F). We also observe the same increase evaluating TNF- $\alpha$ production by lymphocytes and granulocytes, but in monocytes we only observed this increase when comparing Leish-L and Leish-F with the CT group (Fig. 3F). Interestingly, we observed significant differences in the production of most cytokines between the Leish-L and Leish-F groups. This found is very important because it shows that the live parasites are more prone to modify the immunologic environment trough cytokines release.

\subsection{The infection of hHSC by L. Infantum increases in vitro hematopoiesis, favoring erythroid colony formation}

Aiming to evaluate the impact of $L$. infantum infection on hematopoiesis profile, we performed an in vitro hematopoiesis assay, after a short-term hHSC/Leishmania interaction, until 14 days of differentiation. At the end point of the cell differentiation period, we photographed the wells and counted the colonies formed (Fig. 4A-D). A higher number of total colonies were formed when previously stimulated with $L$. infantum (Leish) parasites, compared to the unstimulated cultures (CT) (Fig. 4E). Evaluating separately the white and red colonies, we observed an increased number of red colonies in Leish group in comparison with the CT group. The same increase occurs comparing
A
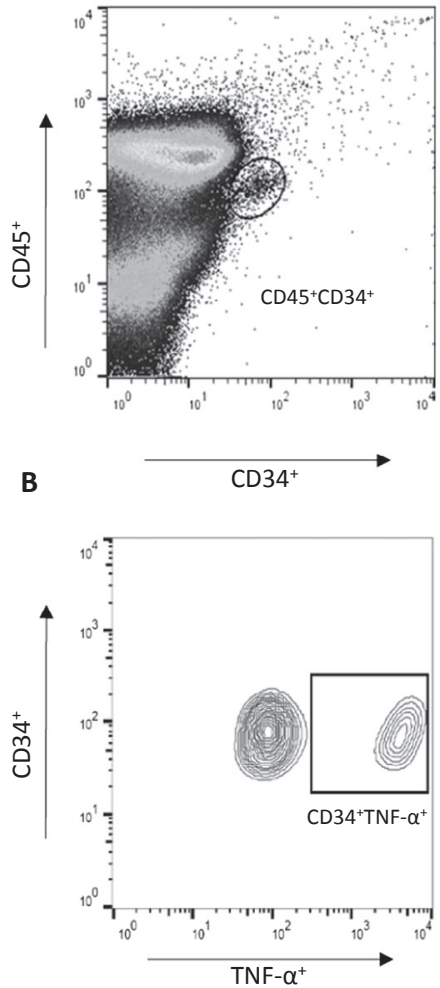
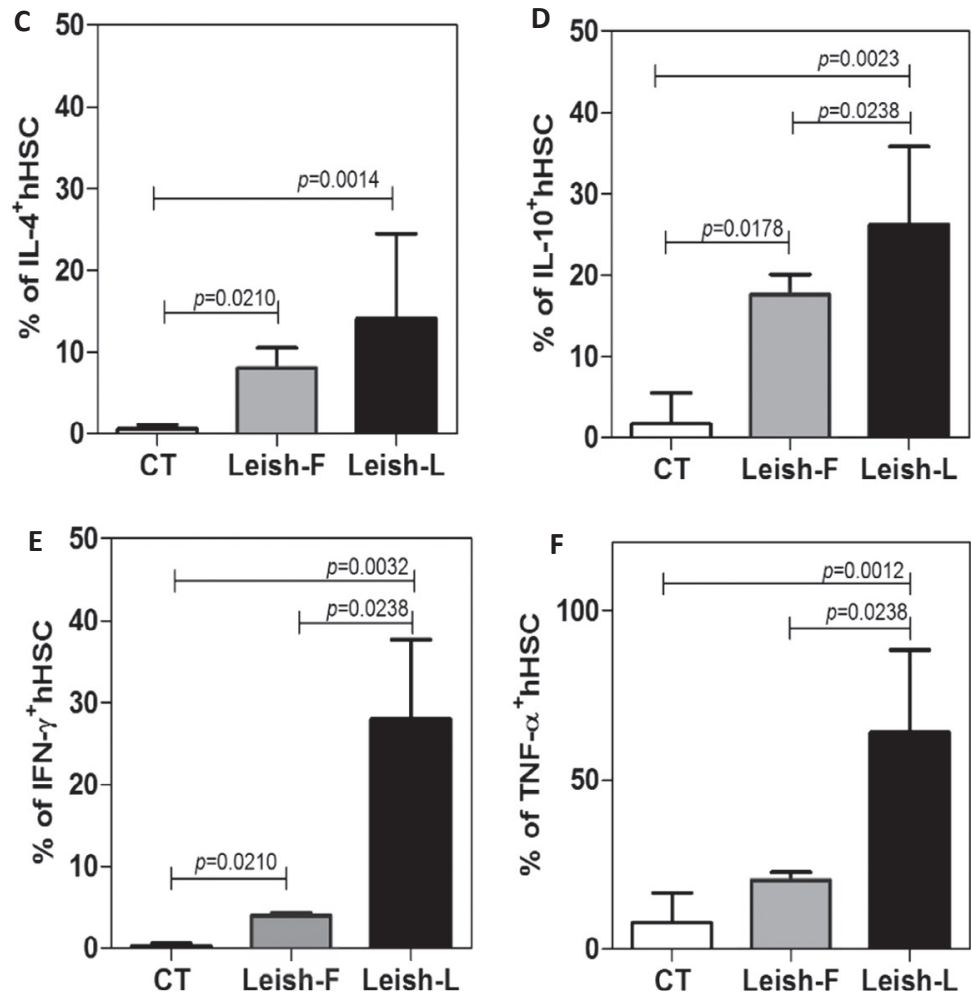

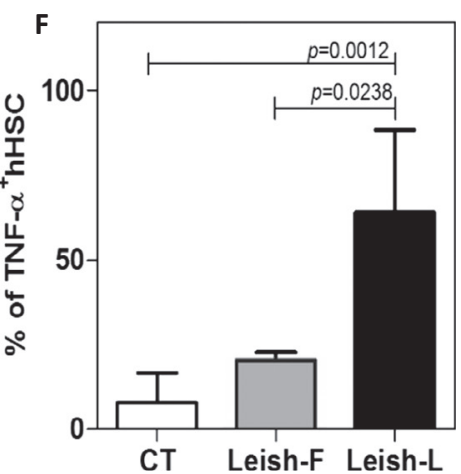



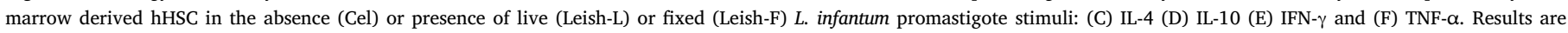
expressed as mean percentage \pm standard error (SE) of $\mathrm{hHSC}^{+}$Cytokine ${ }^{+}$. Significance was considered at $\mathrm{p} \leq 0.05$ and highlighted by lines between the three distinct groups. 
A
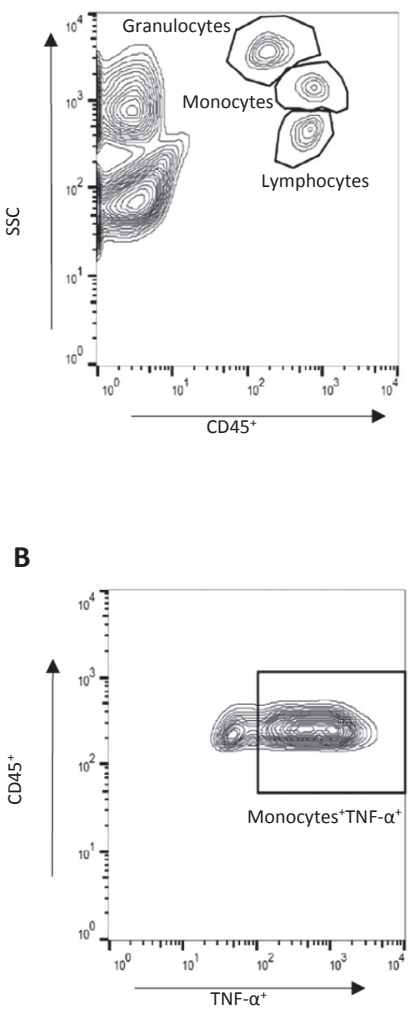

C

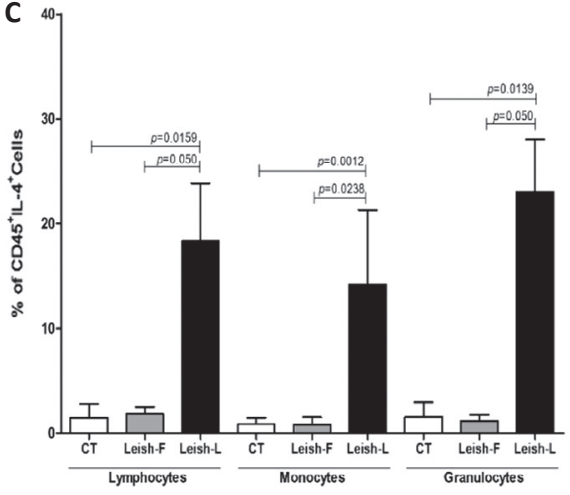

$E$

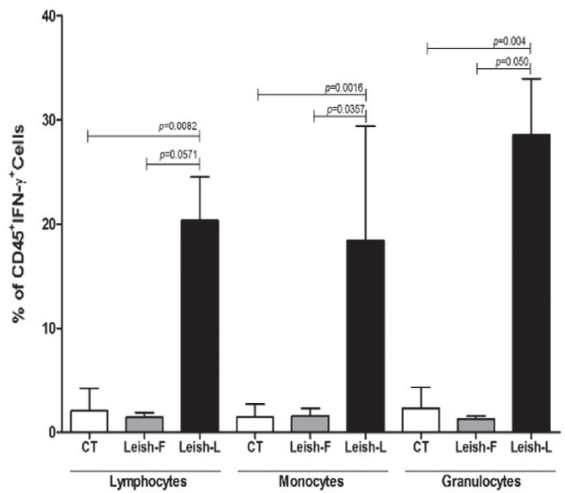

D



$\mathbf{F}$

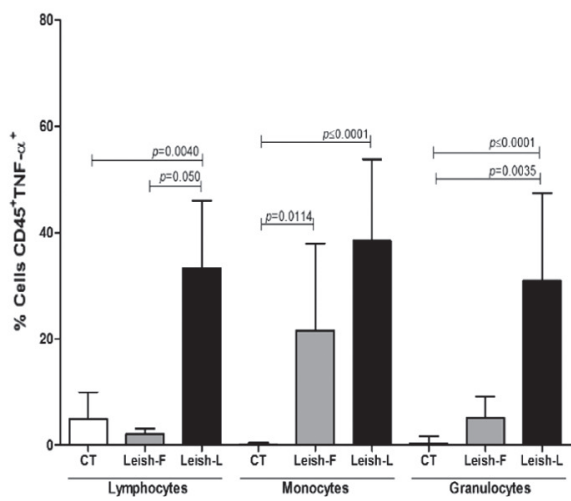

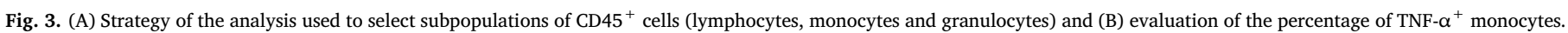

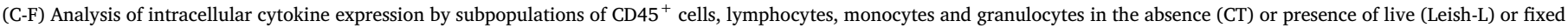

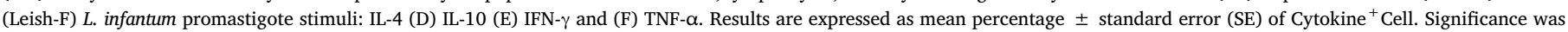
considered at $\mathrm{p} \leq 0.05$ and highlighted by lines between the three distinct groups.

red colonies in Leish group with white colonies in CT group and white colonies in Leish group (Fig. 4F), concluding that L. infantum stimulates erythropoiesis.

\subsection{Infantum infection induces a different pattern of cell differentiation during hematopoiesis affecting surface marker expression after in vitro hematopoiesis}

After ensuring the capacity of hHSC to phagocytize $L$. infantum promastigotes, and the ability to respond to infection through an altered cytokine production and hematopoietic pattern, we compared the possible differences in phenotypic cell profile after $L$. infantum infection. To assess this data, we evaluated surface marker expression at two different time points of infection, at $2 \mathrm{~h}$ (T0) and at 14 days (T14), the final point of hematopoiesis. Thus, when we evaluated the subpopulations of $\mathrm{CD} 45^{+}$cells we observed an increase in the percentage of lymphocytes and granulocytes comparing Leish-L group at T14 with Leish-L group at T0 (Fig. 5B and D). In contrast, we had a diminished percentage of lymphocytes in the CT group compared to the same group after in vitro hematopoiesis (Fig. 5B). Evaluating the percentage of $\mathrm{CD} 45^{+}$monocytes, we found a decrease when comparing the CT group with Leish-L at T0 (Fig. 5C). Moreover, when we evaluated cells of the granulocytic lineage, $\mathrm{CD}_{3} 3^{+}$, and erythrocytic lineage, $\mathrm{CD} 235 \mathrm{a}^{+}$, we observed an increase in the percentage of these cells comparing Leish-L, Leish-F and CT groups after 14 days of culture with the same groups at time T0 (Fig. $5 \mathrm{H}$ and $\mathrm{K}$ ). Besides this, evaluating markers of the monocytic lineages $\mathrm{CD}_{14}{ }^{+}$and HLA-DR we observed a significant increase of HLA-DR MFI in Leish-L group at T0 compared with the CT group and higher percentage of $\mathrm{CD} 14^{+}$cells comparing Leish-L at T14 with Leish-F at the same point in time and CT at T0 (Fig. 5E and F).
When we analyzed $\mathrm{CD} 19^{+}$cells, a marker of lymphocytic lineage, we found an increase of these cells in Leish-L and CT groups at T14 compared with the respective groups at T0 (Fig. 5I). Our data demonstrated no statistical differences when we evaluated CD13 ${ }^{+}$cells and CD41a ${ }^{+}$ cells in all groups at both time points (Fig. $5 \mathrm{G}$ and $\mathrm{J}$ ). Thus, through these data we observed that the infection of hHSC by $L$. infantum in addition to alter the functional profile of infected cells is also capable to increase in vitro hematopoiesis and modify the phenotypic profile of their progenies.

\section{Discussion}

Immunocompromised VL patients exhibit non-typical clinical manifestations that could be difficult to diagnose fast and accurately, leading eventually to patient decease. The absence of positive results in some clinical tests, confusing clinical signs and hematopoietic alterations are the main features of Leishmaniasis after immunossupression. Some studies, mainly case reports, have found Leishmania spp. in sites such as vitreous humour, spleen and bone marrow. These findings show that these parasites seek sites of immunological privilege for their maintenance and multiplication, being the immune system unabled to clear them.

Using murine models, Cotterell et al. [7] had previously demonstrated the capacity of $L$. donovani to infect bone marrow and spleen cells. Additionally, Allahverdiyev et al. [3] found that adipose tissuederived mesenchymal stem cells serve as a host in latent Leishmania infection. In the present study, we had a rare opportunity to evaluate the capacity of $L$. infantum to infect hematopoietic stem cells and to observe the impact of this infection during the process of hematopoiesis in vitro using human samples provided by leukapheresis. 


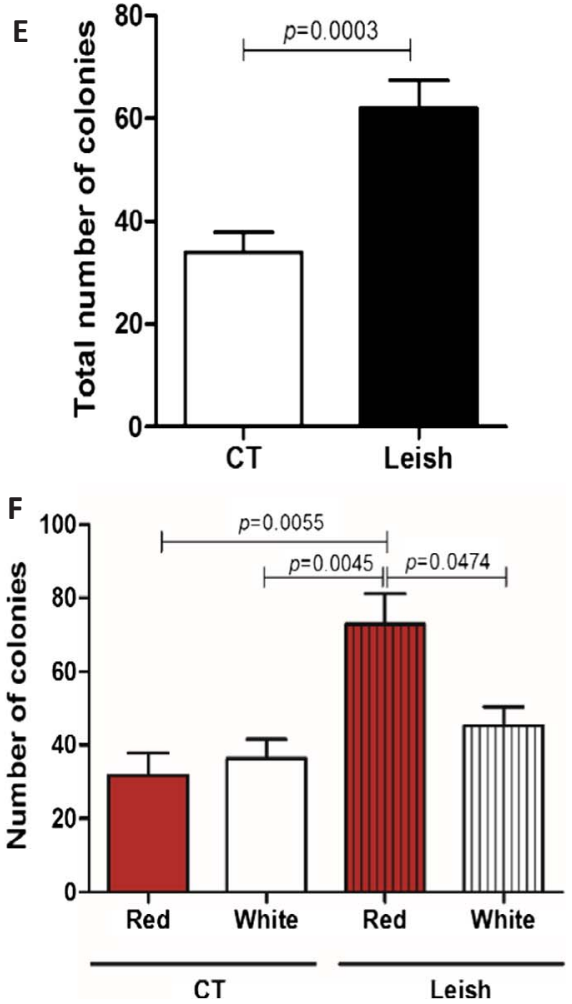

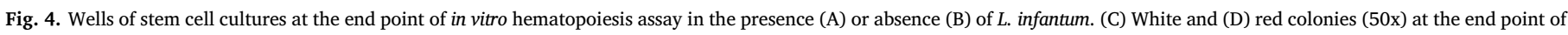



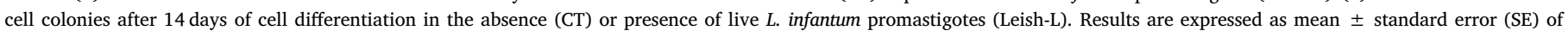
absolute number of colonies. Significance was considered at $\mathrm{p} \leq 0.05$ and highlighted by lines between the three distinct groups.

We showed that human hematopoietic stem cells, monocytes and granulocytes can be infected in vitro with $L$. infantum. The fact that $L$. infantum is able to infect hematopoietic stem cells derived from bone marrow seems to be advantageous for the maintenance of asymptomatic infection, since bone marrow is one of the immunologically privileged sites of the human body. It has been widely reported that one mechanism employed by Leishmania to evade the immune system and remain alive in an infected cell is the blockage or delay of different apoptosis pathways, such as the mitochondrial apoptotic pathway and death triggered by extracellular ATP $[1,10]$. Thus, the process of phagocytosis and the mechanisms triggered by the parasite that guarantee the survival of the parasitized cell are events of great importance that may favor the maintenance of the infection. Studies in murine models point stromal macrophages as the main host cells in chronic LV [7]. This hypothesis is reinforced by the low rate of infection of hematopoietic stem cells in infected animals, suggesting stromal cells as site of replication and maintenance of amastigotes $[7,16]$. In contrast with these results, we found a high frequency of hematopoietic stem cells that phagocytized $L$. infantum promastigotes. We also observed a high percentage of monocytes and granulocytes that phagocytized $L$. infantum. After the internalization of the parasite, we also observed, through the expression of HLA-DR, the activation of monocytes, showing the ability of these cells to respond to the stimuli caused by the presence of Leishmania. Besides the increased frequency of infection, hHSC and subpopulations of $\mathrm{CD}_{4} 5^{+}$cells were able to produce cytokines in response to parasite presence. We observed a great percentage of cells producing important cytokines involved in VL infection, such as IL-10, IL-4, IFN- $\gamma$ and TNF- $\alpha$.

The balance between modulatory and pro-inflammatory cytokines has been identified as a key in the control and healing of VL. We have recently seen that the role played by some cytokines is extremely dependent on the context and microenvironment in which they act. IL-4, for example, has already been related only to anti-inflammatory events, but now it is known that this cytokine participates in synergy with IFN$\gamma$ in the intracellular NO production pathway, an important leishmanicidal mechanism $[17,20,29]$. Interestingly, although NO can kill intracellular parasites, this molecule can play a dual role, being involved in T cells immunosuppression and apoptosis [5,23]. In our results we not only observed a high percentage of hHSC and $\mathrm{CD} 45^{+}$cells producing IL-4, but also a lower percentage of lymphocytes after hematopoiesis of $L$. infantum infected cells. This could represent an important mechanism of regulation because although NO can causes the intracellular death of Leishmania, the same molecule decreases the activity of T lymphocytes, promoting the maintenance of the infection. Schmid and collaborators [24] associated T lymphocyte modulation during L. major infection to MDSCs (Myeloid-derived Suppressor Cells). These cells are derived from myeloid precursors but have been prevented from fully differentiating into mature cells [31]. This cell phenotype was not evaluated in our study, but could be related to some of our findings. MDSCs can be activated and expanded through the stimulation of Toll-like receptors and cytokines such as IFN- $\gamma$ and IL-4, which can trigger pathways such as STAT6, STAT1 and NF-kB [9]. These cells are also able to phagocytize Leishmania, produce ROS and NO and increase the secretion of IL-10, an important modulatory cytokine, in the context of VL [24]. Interesting, we observed an increased percentage of hHSC and $\mathrm{IL}-10^{+} \mathrm{CD} 45^{+}$producer cells, especially granulocytes. Modulation of anti-Leishmania immune response by MDSCs could support the hypothesis of the maintenance of an asymptomatic residual infection in bone marrow cells, with great potential for reactivation in cases of immunosuppressed patients.

Alteration of hematopoiesis is one of most common clinical findings in immunossuppressed patients with VL. Our results demonstrated that the presence of $L$. infantum infected cells alters the hematopoietic profile during hematopoiesis in vitro. We observed a greater number of cell colonies in cultures stimulated with $L$. infantum promastigotes after 14 days. hHSC generally remain in a quiescent or dormant state but can 

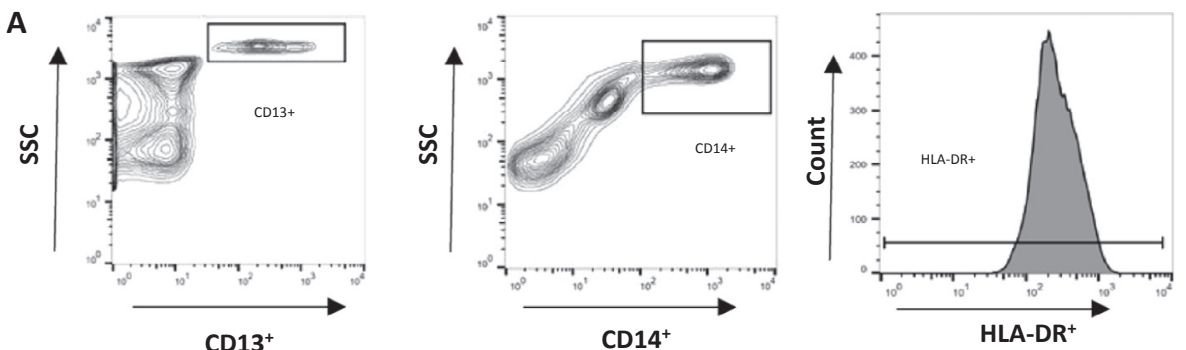

B



D

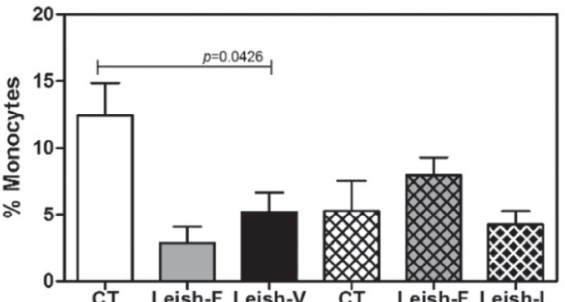

$\mathbf{F}$

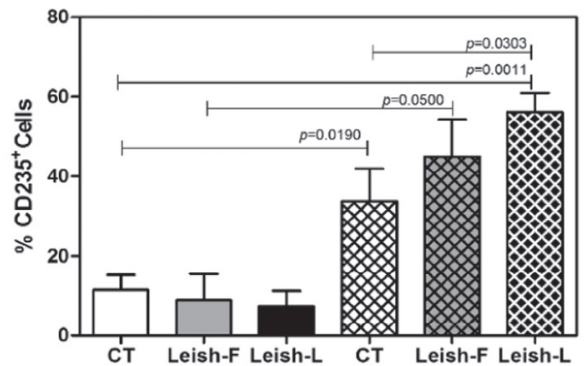

H
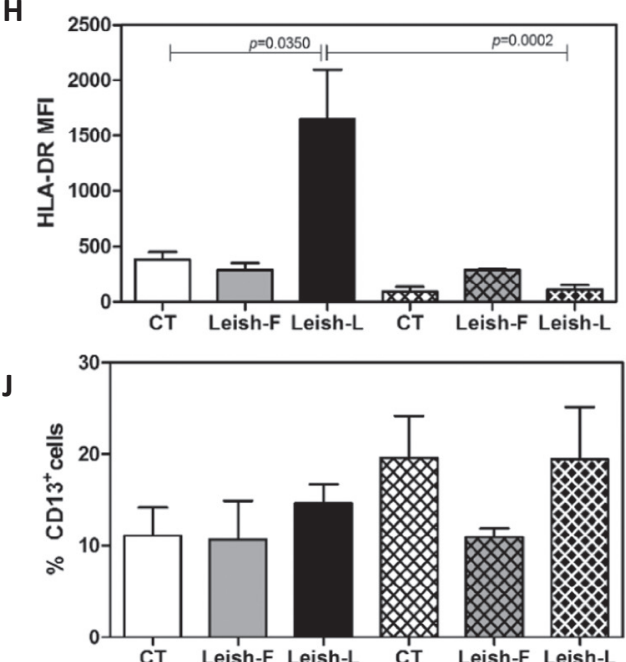

TO

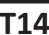

C

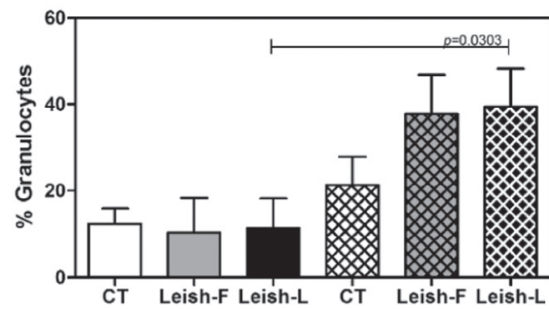

E



G
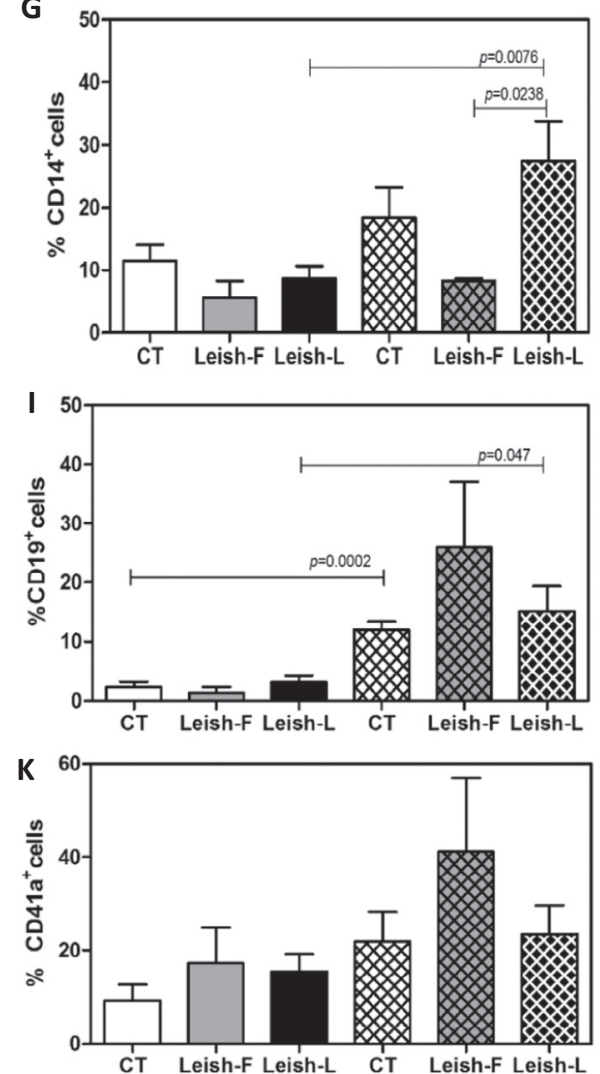

Fig. 5. (A) Strategy of the analysis used to select populations of $\mathrm{CD}_{13}{ }^{+}$and $\mathrm{CD} 14^{+}$cells and to analyze HLA-DR ${ }^{+}$cells by mean fluorescence intensity (MFI). The strategy of the analysis of lymphocytes, granulocytes and monocytes (B, C and D) was showed in Fig. 1B. (B to K) Analysis of the phenotypic profile of bone marrow derived cells by expression of surface markers of granulocytic, lymphocytic, monocytic, erythrocitic and platelet lineages at two different time points - T0 and T14. Cell cultures were not stimulated (CT) or stimulated with live (Leish-L) or fixed (LeishF) L. infantum promastigotes. Results are expressed as mean percentage \pm standard error (SE) or MFI \pm standard error (SE) of each surface marker. Significance was considered at $\mathrm{p} \leq 0.05$ and highlighted by lines between the three distinct groups. 
be stimulated under conditions of stress. They are able to proliferate and differentiate to replenish cellular progeny after cell death or apoptosis [4]. Thus, the presence of Leishmania infected cells could trigger events that could disrupt the hematopoietic balance, thus increasing cell differentiation. According to this hypothesis, a study from Cotterell et al. [7] found elevated levels of GM-CSF and TNF- $\alpha$ mRNA in cell cultures in the presence of $L$. donovani amastigotes and the absence of exogenous growth factors. The role of TNF- $\alpha$ has been extensively investigated in the context of VL as a potent pro-inflammatory molecule. This cytokine can also participate in the regulation of hematopoiesis, yet with a not well defined role $[6,15]$. It is known that, experimentally, $L$. donovani induced-TNF- $\alpha$ acts in synergy with GM-CSF to form CFU-GM, but TNF- $\alpha$ alone had no effect on myelopoiesis [7]. Besides this, TNF- $\alpha$ produced by $\mathrm{CD}^{+}$bone marrow derived cells enhanced HSC clonogenicity, increased the proportion of multipotent progenitors in vitro, and prevented HSC apoptosis in vitro and in vivo [22]. In the present study, we did not quantify GM-CSF, but we observed a high percentage of cells producing TNF- $\alpha$, especially hHSC and monocytes. Furthermore, the augmented frequency of $L$. infantum phagocytosis was still observed in monocytes. Thereby, these data suggest that the production of TNF- $\alpha$ by infected cells could be closely related to increased hematopoiesis.

An increase of myelopoiesis in cultures of $L$. donovani infected cells has previously been reported [7]. The greater number of CFU-GM reinforced this increase after cell culture in the presence of the parasite. In contrast, another interesting finding of our study was the greater number of red colonies in cultures with $L$. infantum infected cells after in vitro hematopoiesis in addition to a greater percentage of CD235a ${ }^{+}$ cells. Our results are supported by the findings of Lafuse and collaborators (2013), who also observed increased frequency of BFU-E and CFU-E after $L$. donovani infection in the gold hamster. It is known that anemia is one of the main clinical findings during developmental of VL. Low erythrocyte and erythrocyte precursor counts were also found in studies with dogs infected with $L$. chagasi $[19,26]$. Thus, the increase in the number of red colonies triggered by the presence of Leishmania seems to be controversial, but this could be explained by a failure of red blood cell differentiation. Steady-state levels of circulating erythrocytes are maintained by erythropoiesis in the bone marrow [25]. Some studies reveal that analysis of bone marrow biopsies of VL patients indicate cellular hyperplasia with many abnormal erythroblasts, which include large erythroblasts containing giant lysosomes and nuclei with little condensed chromatin and multinuclear erythroblasts with irregular nuclei $[13,30]$. Furthermore, a decreased expression of erythroid differentiation genes has been observed alpha-globin, beta-globin, and ALAS2), which are target genes of GATA1 indicating a block in erythroblast differentiation [14]. So, despite the greater numbers of erythroid progenitors, failure of red blood cell differentiation may occur, resulting in anemia in VL patients.

The evaluation of hematopoietic stem cell infection by Leishmania and the consequences of the presence of the parasite in the functional context of the bone marrow are extremely important to understanding peculiar cases of VL in immunocompromised hosts. Additionally, evaluating these aspects using human bone marrow samples is a rare opportunity to better understand $L$. infantum infection dynamics, while the vast majority of the studies are made only on non-human models. Taking our data together, we can conclude that still undifferentiated bone marrow cells are able to phagocytize Leishmania and respond to infection with the production of cytokines. In addition, macrophages and granulocytes are also important phagocytes in the context of infection, becoming activated and responding after parasite internalization. Interestingly, the presence of $L$. infantum induces a differentiated profile of hematopoiesis, favoring the proliferation of cells of the erythroid lineage and altering the phenotypic profiles of their progeny.

\section{Conflict of interest}

The authors declare no conflict of interest.

\section{Acknowledgments}

The authors thank the Flow Cytometry Core Facility (PDTIS/ FIOCRUZ) for flow cytometric acquisition and analysis. This work was supported by Fundação de Amparo à Pesquisa do Estado de Minas Gerais (FAPEMIG) - APQ-000647-13, Conselho Nacional de Desenvolvimento Científico e Tecnológico (CNPq) by fellowship awarded to LRVA, OAMF and ATC. RCG received fellowship from CNPq program. DM is supported by a $\mathrm{PhD}$ fellowship SFRH/BD/91543/2012, MR is supported by a PhD fellowship SFRH/BD/89871/2012. RS is supported by FCT Investigator 2014 (IF/00021/2014).

\section{References}

[1] K. Akarid, D. Arnoult, J. Micic-Polianski, J. Sif, J. Estaquier, J.C. Ameisen, Leishmania major-mediated prevention of programmed cell death induction in infected macrophages is associated with the repression of mitochondrial release of cytochrome c, J. Leukoc. Biol. 76 (2004) 95-103.

[2] J. Alexander, D.G. Russell, The interaction of Leishmania species with macrophages, Adv. Parasitol. 31 (1992) 175-254.

[3] A.M. Allahverdiyev, M. Bagirova, S. Elcicek, R.C. Koc, S.Y. Baydar, N. Findikli, O.N. Oztel, Adipose tissue-derived mesenchymal stem cells as a new host cell in latent leishmaniasis, Am. J. Trop. Med. Hyg. 85 (2011) 535-539.

[4] M.T. Baldridge, K.Y. King, M.A. Goodell, Inflammatory signals regulate hematopoietic stem cells, Trends Immunol. (2011).

[5] V. Bronte, P. Zanovello, Regulation of immune responses by L-arginine metabolism, Nat. Rev. Immunol. 5 (2005) 641-654.

[6] H.E. Broxmeyer, D.E. Williams, The production of myeloid blood cells and their regulation during health and disease, Crit. Rev. Oncol. Hematol. 8 (1988) 173-226.

[7] S.E. Cotterell, C.R. Engwerda, P.M. Kaye, Enhanced hematopoietic activity accompanies parasite expansion in the spleen and bone marrow of mice infected with Leishmania donovani, Infect. Immun. 68 (2000) 1840-1848.

[8] J.S. Fisher, E.S. Woodle, J.R. Thistlethwaite, Kidney transplantation: graft monitoring and immunosuppression, World J. Surg. 26 (2002) 185-193.

[9] D.I. Gabrilovich, S. Nagaraj, Myeloid-derived suppressor cells as regulators of the immune system, Nat. Rev. Immunol. 9 (2009) 162-174.

[10] B.K. Kolli, J. Kostal, O. Zaborina, A.M. Chakrabarty, K.P. Chang, Leishmania-released nucleoside diphosphate kinase prevents ATP-mediated cytolysis of macrophages, Mol. Biochem. Parasitol. 158 (2008) 163-175.

[11] C.N. Kotton, Zoonoses in Solid-Organ and Hematopoietic Stem Cell Transplant Recipients, Clin. Infect. Dis. 44 (2007) 857-866.

[12] A.M. Krensky, Immunologic tolerance, Pediatr. Nephrol. 16 (2001) 675-679.

[13] P.V. Kumar, M. Vasei, A. Sadeghipour, E. Sadeghi, H. Soleimanpour, A. Mousavi, A.H. Tabatabaei, M.M. Rizvi, Visceral leishmaniasis: bone marrow biopsy findings, J. Pediatr. Hematol. Oncol. 29 (2007) 77-80.

[14] W.P. Lafuse, R. Story, J. Mahylis, G. Gupta, S. Varikuti, H. Steinkamp, S. Oghumu, A.R. Satoskar, Leishmania donovani Infection induces anemia in hamsters by differentially altering erythropoiesis in bone marrow and spleen, PLoS One 8 (2013).

[15] F. Lardon, H.W. Snoeck, Z.N. Berneman, V.F. Van Tendeloo, G. Nijs, M. Lenjou, E. Henckaerts, C.J. Boeckxtaens, P. Vandenabeele, L.L. Kestens, D.R. Van Bockstaele, G.L. Vanham, Generation of dendritic cells from bone marrow progenitors using GM-CSF, TNF-alpha, and additional cytokines: antagonistic effects of IL-4 and IFN-gamma and selective involvement of TNF-alpha receptor-1, Immunology 91 (1997) 553-559.

[16] V. Leclercq, M. Lebastard, Y. Belkaid, J. Louis, G. Milon, The outcome of the parasitic process initiated by Leishmania infantum in laboratory mice: a tissuedependent pattern controlled by the Lsh and MHC loci, J. Immunol. 157 (1996) 4537-4545.

[17] M.D. Mossalayi, M. Arock, D. Mazier, P. Vincendeau, I. Vouldoukis, The human immune response during cutaneous leishmaniasis: NO problem, Parasitol. Today 15 (1999) 342-345.

[18] A.T. Nguyen Hoang, H. Liu, J. Juaréz, N. Aziz, P.M. Kaye, M. Svensson, Stromal cellderived CXCL12 and CCL8 cooperate to support increased development of reg ulatory dendritic cells following Leishmania infection, J. Immunol. 185 (4) (2010) 2360-2371.

[19] R.D.C. Nicolato, R.T. De Abreu, B.M. Roatt, R.D.D.O. Aguiar-Soares, L.E.S. Reis, M.D.G. Carvalho, C.M. Carneiro, R.C. Giunchetti, L.E.M. Bouillet, D.S. Lemos, W. Coura-Vital, A. Barbosa Reis, Clinical forms of canine visceral leishmaniasis in naturally Leishmania infantum-infected dogs and related myelogram and hemogram changes, PLoS One 8 (2013).

[20] N. Paul-Eugène, J.-P. Kolb, M. Sarfati, M. Arock, H. Quaaz, P. Debré, D.M. Mossalayi, B. Dugas, Ligation of CD23 activates soluble guanylate human monocytes cyclase in human monocytes via an L-arginine-dependent mechanism, 57 (1995) 160-167.

[21] R.D. Pearson, A.Q. Sousa, Clinical spectrum of Leishmaniasis, Clin. Infect. Dis. 22 (1996) 1-13. 
[22] F. Rezzoug, Y. Huang, M.K. Tanner, M. Wysoczynski, C.L. Schanie, P.M. Chilton, M.Z. Ratajczak, I.J. Fugier-Vivier, S.T. Ildstad, TNF-alpha is critical to facilitate hemopoietic stem cell engraftment and function, J. Immunol. 180 (2008) 49-57.

[23] P.C. Rodríguez, A.C. Ochoa, Arginine regulation by myeloid derived suppressor cells and tolerance in cancer: Mechanisms and therapeutic perspectives, Immunol. Rev. 222 (2008) 180-191.

[24] M. Schmid, N. Zimara, A.K. Wege, U. Ritter, Myeloid-derived suppressor cell functionality and interaction with Leishmania major parasites differ in C57BL/6 and BALB/c mice, Eur. J. Immunol. 44 (2014) 3295-3306.

[25] U. Testa, Apoptotic mechanisms in the control of erythropoiesis, Leukemia 18 (2004) 1176-1199.

[26] R. Tropia de Abreu, M. Carvalho, C.M. Carneiro, R.C. Giunchetti, A. TeixeiraCarvalho, O.A. Martins-Filho, W. Coura-Vital, R. Correa-Oliveira, A.B. Reis, Influence of clinical status and parasite load on erythropoiesis and leucopoiesis in dogs naturally infected with leishmania (Leishmania) chagasi, PLoS One 6 (2011) e18873.
[27] J. Van Griensven, E. Carrillo, R. López-Vélez, L. Lynen, J. Moreno, Leishmaniasis in immunosuppressed individuals, Clin. Microbiol. Infect. 20 (2014) 286-299.

[28] D.M. Vitelli-Avelar, R. Sathler-Avelar, A.P.B. Wendling, R.D.R. Rocha, A. TeixeiraCarvalho, N.É. Martins, J.C.P. Dias, A. Rassi, A.O. Luquetti, S.M. Elói-Santos, O.A. Martins-Filho, Non-conventional flow cytometry approaches to detect antiTrypanosoma cruzi immunoglobulin G in the clinical laboratory, J. Immunol. Methods 318 (2007) 102-112.

[29] I. Vouldoukis, V. Riveros-morenot, B. Dugas, F. Ouaaz, P. Becherel, P. Debre, S. Moncadat, M.D. Mossalayi, The killing of Leishmania major by human macrophages is mediated by nitric oxide induced after ligation of the FccRII / CD23 surface antigen, Proc. Natl. Acad. Sci. 92 (1995) 7804-7808.

[30] S.N. Wickramasinghe, R.E. Phillips, S. Looareesuwan, D.A. Warrell, M. Hughes, The bone marrow in human cerebral malaria: parasite sequestration within sinusoids, Br. J. Haematol. 66 (1987) 295-306.

[31] J.-I. Youn, S. Nagaraj, M. Collazo, D.I. Gabrilovich, Subsets of myeloid-derived suppressor cells in tumor-bearing mice, J. Immunol. 181 (2008) 5791-5802. 